\section{SAT0157 THE SEX HORMONE BINDING GLOBULIN IN MALE OSTEOPOROSIS}

ER Legrand, C Gibert, Y Gallois, E Mathieu, F Boux de casson, MF Basle, D Chappard, M Audran. Service de Rhumatologie, Centre Hospitalier Universitaire, Angers, France

\subsection{6/annrheumdis-2001.644}

Background Several studies have shown that the increase in Sex Hormone Binding Globulin and the decrease in biovailable estradiol and testosterone can explain age-induced bone loss in healthy men over 70 years.

Objectives To evaluate the role of the Sex Hormone Binding Globulin (SHBG) in the pathophysiology of osteoporosis in middle-aged men.

Methods 80 men with osteoporosis (T-score $<-2.5$ ) and 40 agematched controls were recruited to evaluate the relationships between sex hormone levels, bone markers levels, bone mineral density and vertebral fractures. Fasting serum samples were assayed for total and free testosterone, total estradiol and SHBG. Bone remodelling was evaluated by measurement of urinary levels of the C-telopeptide of type I collagen (CTX) and free deoxypyridinoline (D-Pyr), serum osteocalcin and bone specific alkalin phosphatase (BSAP).

Results

- There was no difference between controls and osteoporotic men according to age, BMI, total testosterone and estradiol. In contrast, serum SHBG level was significantly higher (+ $42.2 \%)$ whereas free androgen index was lower (- 24.8\%) in patients with osteoporosis.

- Stepwise linear regression analysis showed that SHBG was significantly correlated with D-Pyr $(r=0.33)$, CTX $(r=$ $0.25)$ and BSAP $(r=0.30)$. (3) After adjustment for age and BMI, hip BMD was not associated with total testosterone or estradiol but only with serum SHBG $(r=-0.35)$. (4) Among osteoporotic patients, spinal radiographs evidenced at least one vertebral crush fracture in 36 men and none in 44 patients. Serum SHBG concentration was significantly associated with the presence of vertebral fracture: odds ratio was 2.0 (95\% CI: 1.2-3.5) for an increase of one standard deviation of SHBG.

Conclusion The present study showed that serum SHBG concentration is increased in most middle-aged men with primary or secondary osteoporosis and is correlated with bone remodelling markers, hip bone mineral density and vertebral fracture risk. These results might represented a plausible explanation of bone loss in young and middle-aged men with idiopathic osteoporosis and vertebral fractures.

\section{SAT0158 ASSOCIATES WITH AND EXTENT OF REDUCED BONE MINERAL DENSITY (BMD) IN FEMALE RHEUMATOID ARTHRITIS (RA) PATIENTS: THE OSLO, TRURO, AMSTERDAM (OSTRA) COLLABORATIVE STUDY}

${ }^{1} \mathrm{G}$ Haugeberg, ${ }^{2} \mathrm{M}$ Lodder, ${ }^{2} \mathrm{~W}$ Lems, ${ }^{1} \mathrm{~T}$ Uhlig, ${ }^{2} \mathrm{~B}$ Dijkmans, ${ }^{1} \mathrm{~T}$ Kvien, ${ }^{3} \mathrm{~A}$ Woolf. ${ }^{1}$ Department of Rheumatology, Diakonhjemmet Hospital, Oslo, Norway; ${ }^{2}$ Department of Rheumatology, Vrije Universiteit, Amsterdam, Netherlands; ${ }^{3}$ Department of Rheumatology, Royal Cornwall Hospital, Truro, UK

\subsection{6/annrheumdis-2001.645}

\section{Background}

Objectives To examine associates with and occurrence of reduced $\mathrm{BMD}$ in female RA patients from three European countries.
Methods 150 (50 from each country) consecutive female RA patients (ACR 1987 criteria, disease duration $\geq 5$ yrs, age 50-70 yrs), visiting the out-patient rheumatology clinics were included. Study variables included clinical data, laboratory tests, hand Xray (Larsen score) and BMD measurement. BMD $\left(\mathrm{g} / \mathrm{cm}^{2}\right)$ was measured by dual energy X-ray absorptiometry (DXA) and adjusted for age, weight and height. A pooled European/US reference population, showing no substantial differences with published Norwegian, English, and Dutch reference populations, was used to calculate T-scores. Groups were compared by one way ANOVA and cross tabulation (Pearson Chi square test). To investigate associates for BMD reduction bi- and multi-variety statistical analyses were performed.

Results No significant differences between the countries were found for age, disease duration and rheumatoid factor, whereas weight and most disease related variables, including the use of prednisolone and treatment for osteoporosis, differed between the 3 groups. Percentage of patients having osteoporosis (T-score $<-2.5 \mathrm{SD})$ at femoral neck was $21.3 \%$ in Oslo, $8.3 \%$ in Truro, and $18.4 \%$ in Amsterdam $(\mathrm{P}=0.19)$ and at the spine $32.0 \%$, $18.4 \%$ and $12.0 \%(\mathrm{P}=0.04)$, respectively. Mean $(\mathrm{SD}) \mathrm{BMD}$ values are shown in the Table 1 . Using multivariate linear regression age, weight, total hand X-ray Larsen score, previous history of clinical fracture and ever use of DMARDs were independent associates for femoral neck BMD. For spine L2-4 weight, mean CRP last 12 months, ever use of DMARDs and current use of prednisolone were independent associates.

\begin{tabular}{lllll}
\multicolumn{6}{l}{ Abstract SAT0158 Table 1 } & & \\
\hline & Oslo & Truro & Amsterdam & P-value \\
\hline Fem.neck & $0.80(0.06)$ & $0.86(0.10)$ & $0.83(0.07)$ & 0.001 \\
Spine L2-4 & $1.06(0.06)$ & $1.12(0.12)$ & $1.09(0.09)$ & 0.002 \\
\hline
\end{tabular}

Conclusion Differences in femoral neck and spine BMD might reflect differences in life styles factors, disease activity and disease management between the countries.

\section{SAT0159 NON-VERTEBRAL FRACTURES AND ITS ASSOCIATES IN FEMALE RHEUMATOID ARTHRITIS (RA) PATIENTS: THE OSLO, TRURO, AND AMSTERDAM (OSTRA) COLLABORATIVE STUDY}

${ }^{1} \mathrm{G}$ Haugeberg, ${ }^{2} \mathrm{M}$ Lodder, ${ }^{2} \mathrm{~W}$ Lems, ${ }^{1} \mathrm{R}$ Ørstavik, ${ }^{2} \mathrm{~B}$ Dijkmans, ${ }^{1} \mathrm{~T} K \mathrm{~K}$ ${ }^{1}$ Department of Rheumatology, Diakonhjemmet Hospital, Oslo, Norway; ${ }^{2}$ Department of Rheumatology, Vrije Universiteit, Amsterdam, Netherlands; ${ }^{3}$ Department of Rheumatology, Royal Cornwall Hospital, Truro, UK

\subsection{6/annrheumdis-2001.646}

\section{Background}

Objectives To examine non-vertebral fractures (N-VF) in RA patients, of similar age, disease duration and rheumatoid factor status recruited from three European countries, and their association with bone mineral density (BMD), demographic and disease related associates.

Methods 150 (50 from each country) consecutive female RA patients (ACR 1987 criteria, disease duration $>5$ yrs, age 5070 yrs) visiting the out-patient Department of Rheumatology in the participating centres were included. Data on N-VF occurring after age 25 and after RA diagnosis, demographics and measures of disease activity were collected. Dual energy X-ray 